\title{
LA RELATIVITÀ E LA REALTÀ DEL MOMENTO PRESENTE ${ }^{1}$
}

\author{
MAURO DORATO (*)
}

SuNTO. - In questo saggio discuto il problema del realismo scientifico dal punto di vista della questione del realismo temporale. A questo scopo, esamino quattro diverse teorie sulla realtà del tempo, che corrispondono molto da vicino a quattro posizioni dibattute nell'ambito della discussione sul realismo scientifico, ovvero il realismo sulle teorie, il realismo sulle entità, il realismo strutturale e quello che potremmo chiamare "realismo debole" dato validità intersoggettiva delle teorie. Concludo affermando che il carattere relazione dell' "essere presente" non implica necessariamente la riducibilità degli stati mentali a quelli fisici, ma piuttosto la necessità di considerare come fortemente correlate la successione locale di eventi fisici e la nostra consapevolezza di tale successione dovuta all'integrazione temporale operata dal nostro cervello.

$$
* * *
$$

ABSTRACT. - In this paper I examine the problem of scientific realism from the viewpoint of the question of reality of time, and of the present moment in particular. For this purpose, I examine four different views about the reality of time, corresponding in the debate on scientific realism to theory realism, entity realism, structural realism and what we could call "weak realism" (intersubjectice agreement). I conclude that the relational character of "being present" need not entail the reducibility of mental states to physical states, but rather the necessity of considering the objective succession of physical events perceived by our brain as strongly correlated with the awareness of this succession made possible by our brain.

\section{INTRODUZIONE}

Il problema del realismo, e in particolare di quello cosiddetto "scientifico", è talmente vasto che conviene restringere almeno un po'

1 Ringrazio Matteo Morganti per un'attenta lettura di una bozza di questo saggio.

(*) Dipartimento di Filosofia, Comunicazione e Spettacolo, Università degli Studi “Roma Tre”, Roma, Italia. E-mail: mauro.dorato@uniroma3.it 
la nostra prospettiva partendo da alcune questioni attinenti al problema della "realtà del tempo". ${ }^{2}$ Una discussione ancorché breve su quest'ultimo problema offre infatti un punto di vista privilegiato per cercare di comprendere che cosa significhi più in generale il "realismo", uno degli "ismi" più abusato dai filosofi. Che i due tipi di realismo siano strettamente correlati è stato sostenuto da molti filosofi del tempo: "if we seek to examine Time, we find ourselves examining Reality". (Gunn 1929, p. 369). Negare la realtà del tempo sembra implicare immediatamente la negazione della realtà del mondo esterno, tanto è fondamentale il ruolo che la nozione di tempo svolge nella esperienza soggettiva: se questa non desse informazioni attendibili su ciò che è là fuori, avremmo ragione di dubitare dell'esistenza di tutto ciò che percepiamo.

Una volta operata la scelta di studiare il problema del realismo partendo dal tempo, ci troviamo tuttavia di fronte a profonde aporie, che erano già state evidenziate da Aristotele: "In primo luogo, è bene formulare le aporie relative al tempo, se sia da annoverare tra le cose che sono o tra quelle che non sono, e quindi quale ne sia la natura". Fisica, 217b29.' Tali aporie, afferma Aristotele, derivano non solo dal fatto che, in generale, non è chiaro il rapporto tra tempo e movimento, ma anche dalla difficoltà ad attribuire realtà a qualcosa che è costituito da parti (passato e futuro) che sono irreali. Un tutto esistente/reale non può avere parti irreali.

Per cercare di approfondire nel breve spazio di questo saggio il problema posto da Aristotele, la questione centrale sulla quale mi soffermerò in ciò che segue ha proprio a che fare con il problema dello statuto ontologico di passato e futuro relativamente al momento presente. Esiste infatti un importante conflitto nel nostro modo di rappresentare il tempo, in particolare quello che viene generato dall'accostamento dell'immagine del tempo della nostra esperienza a quella postulata dalla fisica. Se il tempo fisico fosse incompatibile con le caratteristiche essenziali del tempo della nostra esperienza, dovremmo con ciò concludere che quest'ultimo ha un carattere irreale o illusorio?

Nella nostra esperienza soggettiva e certamente per il senso comune, sembra esistere in modo "pieno" ed essere reale solo il presen-

2 Barbour (1999) è un fisico che nega la realtà del tempo, ultimo di una lunga serie di fisici e filosofi che provano a sostenere che il tempo è un'illusione antropomorfica.

3 La traduzione del testo greco è di Ruggio (2008). 
te e gli eventi ad esso simultanei, dove "simultanei" si riferisce anche a eventi arbitrariamente distanti gli uni dagli altri. In una parola, per il senso esiste un presente cosmicamente inteso, come anche Newton riteneva: "we understand any moment of duration to be diffused throughout all spaces, after its own way [unumquodque durationis momentum...per universa spatia, suo more... diffundi intelligimus]" (Hall and Hall 1962, p. 104, quoted in Torretti 2006, p. 9). Gli eventi passati invece vivono solo come tracce nella nostra memoria e nella realtà presente circostante, ed è proprio la fondamentale differenza fenomenologica tra un ricordo e una percezione che ci convince del fatto che ciò che esperiamo nel presente ha - in un senso da chiarire - una "realtà" diversa da ciò che è accaduto nel passato. Quando affiora alla mia memoria un ricordo di evento passato, l'atto del ricordare avviene sempre nel momento presente e si riferisce a un evento ad esso precedente. La consapevolezza immediata dell'esistenza di un rapporto di precedenza temporale tra una rammemorazione presente e il suo contenuto passato è talmente indubitabile che non può non essere messa alla base di qualunque costruzione filosofica o fenomenologica sul tempo.

Strettamente collegata a questa consapevolezza immediata è l'inferenza in base alla quale gli eventi esperiti nel passato sono concepiti come cause di quelli che ora ricordiamo. Più generalmente, gli eventi passati sono cause che spiegano le tracce presenti nel mondo esterno e interno che hanno lasciato dietro di sé. Tuttavia, il mammut vivente in passato il cui scheletro è oggi osservabile come suo successore causale non è parte di ciò che si potrebbe chiamare "un elenco di tutto ciò che esiste", anche se, essendo esistito in passato, ha una natura ontologica diversa da quella di un ente fittizio come Bellerofonte. Per parte loro, gli eventi futuri (il prossimo Natale) sono anticipati nel presente sulla base delle esperienze passate. Essendo almeno in minima parte effetti delle nostre azioni presenti, essi sono tipicamente concepiti come "irreali" o non esistenti e quindi come suscettibili di venire in essere o divenire reali, in minima parte anche grazie a quel che facciamo. Nella nostra esperienza, gli eventi futuri sono quindi visti come un insieme di potenzialità o di possibilità da noi immaginate o previste che si possono attualizzare in un presente di là da venire, ma solo in funzione di ciò che esiste ora e di regolarità osservate in passato che rendono prevedibile il loro corso futuro.

Oltre al carattere ontologicamente privilegiato del presente rispetto al passato e al futuro, già messo in luce da Aristotele e prima ancora da Parmenide - che negava la realtà del tempo perché non ci si 
può riferire a passato e futuro, visto che non sono - la nostra esperienza del tempo genera la credenza indubitabile che il tempo "passi". Questo non significa che tale passaggio si debba interpretare alla lettera come il movimento di un fiume o quello di una torcia che illumini successivamente tutti i momenti del tempo. ${ }^{4}$ Esso tuttavia fa riferimento al fatto indubitabile che ogni giorno, ogni mese e ogni anno ci allontaniamo dal giorno della nostra nascita rispettivamente di un giorno, di un mese e di un anno. Il "tempo di attesa" (waiting time), considerato da Torretti come una delle caratteristiche essenziali del tempo (Torretti 2007, 734) è strettamente legato alla nostra esperienza del suo "passare", che per esempio viene percepito assai lento quando aspettiamo che venga il nostro turno in una lunga fila. Il fatto che la valutazione della durata del tempo in questi casi varii con la nostra impazienza non implica certo che l'esperienza dell'attesa sia illusoria!

Nel tempo fisico, invece, in virtù del fatto che è quantomeno controverso sostenere che le leggi ci permettano di individuare un presente privilegiato, sembra anche difficile giustificare la tesi che il tempo passi, almeno se tale "passare" consiste nel "divenire reale" da parte di un evento o di uno stato di cose che era prima solo possibile, irreale o inesistente. Come vedremo meglio in seguito, da un punto di vista fisico il tempo sembra essere costituito da una successione temporale locale (non estesa ai confini dell'universo) e priva di un presente privilegiato che "scorra".

Considerando il conflitto tra queste due rappresentazioni del tempo, sorge spontanea una domanda fondamentale e genuinamente filosofica. Il suo carattere filosofico è dovuto alla sua generalità e al suo carattere transdisciplinare e quindi al fatto che essa non può riguardare una singola scienza, come la psicologia del senso comune o la fisica, e nemmeno soltanto la fenomenologia della coscienza interna del tempo (à la Husserl). In una parola tale domanda si può porre come segue: che rapporto c'è tra la rappresentazione fisica del tempo e quella della nostra esperienza?

Le risposte che si possono anticipare sono due. La prima si appoggia a una tesi monistica - anche se non necessariamente riduzionistica -, in base alla quale le proprietà temporali fondamentali sono

4 Questa successione del passaggio del tempo visto come analogo, o addirittura identico a, una torcia in movimento è stata recentemente difesa da un metafisico e filosofo della scienza americano (Skow 2015). 
quelle descritte dalla fisica, anche se quelle esperienziali sono irriducibili al tempo fisico perché descritte con linguaggi incommensurabili. La seconda risposta insiste invece sull'inevitabile pluralismo delle rappresentazioni del tempo: non un unico tempo ma "molti tempi", nessuno dei quali caratterizzabile come avente priorità ontologica sugli altri.

$\grave{E}$ importante notare che entrambi i punti di vista (il secondo in modo particolare) si orientano in modo deciso verso l'incompletezza dell'immagine fisica del tempo: esistono genuine proprietà temporali di carattere soggettivo che non sono meno fondamentali o "reali" di quelle oggettive descritte dalla fisica. Indipendente dal difficile tentativo di stabilire quali fossero le opinioni di Einstein sulla natura del presente, nel passo seguente si ha un'enunciazione chiara della tesi dell'incompletezza del tempo fisico: "Once Einstein said that the problem of the Now worried him seriously. He explained that the experience of the Now means something special for man, something essentially different from the past and the future, but that this important difference does not and cannot occur within physics. That this experience cannot be grasped by science seemed to him a matter of painful but inevitable resignation." (Carnap 1963, pp. 37-38). È ironico osservare che, come vedremo meglio in seguito, molta della filosofia del tempo contemporanea utilizza proprio la fisica relativistica per mostrare che l'esperienza del passaggio è illusoria.

Nel prosieguo vorrei presentare degli argomenti - che qui non possono essere pienamente sviluppati - che hanno lo scopo di mostrare che l'esperienza umana del presente e del suo "passare" è irriducibile alla fisi$\mathrm{ca}$, ma che non per questo è "illusoria", in quanto nasce dalla relazione tra successioni di eventi fisici di tipo fra loro assai diverso. Tale relazione lega il nostro apparato cognitivo - e quindi una successione di stati mentali inerenti al nostro corpo - a una successione fisica locale di eventi percepiti, dove "locale" si riferisce tecnicamente a una successione che vale oggettivamente (intersoggettivamente) solo per quegli eventi che sono connettibili da un segnale che viaggi a velocità inferiori o uguali a quelli della luce.

In particolare, il prossimo capitolo del saggio $(\$ 2)$ cercherà di far luce su alcune trappole concettuali nelle quali cadono a volte i filosofi del tempo, che insistono sull'aspetto ontologico del dibattito, ovvero sul fatto che il senso comune attribuisca al futuro e al passato uno statuto di "irrealtà". Ma i riferimenti a realtà e irrealtà sono carichi di fraintendimenti e conducono, se non utilizzati con la dovuta cautela, a pseudo-problemi. Come si vedrà nel capitolo $\mathbb{3}$ questo è evidente 
all'interno del dibattito fra tre teorie metafisiche che hanno profondamente influenzato la filosofia analitica del tempo del secolo scorso. Tale dibattito, con un certo grado di approssimazione, è riassumibile nello scontro fra tre diverse tesi ontologiche, quella in base alla quale esiste solo il presente (presentismo), quella che sostiene che esistono solo il passato e il presente (possibilismo), e quella che consiste nell'affermare che tra passato presente e futuro non v'è alcuna differenza ontologica (eternalismo). Tipicamente, l'ultima posizione è considerata incompatibile con una concezione dinamica del tempo, in base alla quale il venire in essere di eventi futuri è condizione necessaria e sufficiente al passaggio del tempo.

Dopo aver illustrato brevemente gli argomenti in base ai quali la relatività implicherebbe una concezione eternalista, ${ }^{5}$ in cui il mutamento è dato dal fatto che un ente (identificato da relazioni che garantiscono una continuità causale) esemplifica in istanti di tempo diversi proprietà incompatibili, chiuderò poi il saggio mostrando come la fenomenologia dell'esperienza del presente non possa essere ridotta alla descrizione di una sequenza di eventi fornita dalla fisica $(\$ 4)$

\section{CHE COSA SIGNIFICA REALISMO?}

Cominciamo con il notare che l'uso del predicato (?) "è reale", come altri predicati spesso oggetto di indagini filosofiche, dovrebbe sempre implicare l'esistenza di quella che chiamerò una classe di contrasto con qualcosa che conta come "irreale". Per esempio, non si dà illusione senza qualcosa che non è un'illusione, né un dubbio senza una credenza di cui non dubitiamo. Se tutte le nostre percezioni fossero illusorie, rispetto a quali altre percezioni avremmo ragione a sostenere una tesi del genere?

Allo stesso modo, l'aggettivo "reale" in "il presente è una proprietà reale degli eventi" necessita di una classe di contrasto tra "reale" e "irreale", in modo analogo a espressioni del tipo "questo è vero caffè e non un surrogato", "questo è un treno reale e non un treno-giocattolo", questo è DNA reale ("vero DNA") e non un mero modello. Come si esprimeva Austin in contesti linguistici analoghi con un'espressione non politica-

5 Per una trattazione più dettagliata di questi argomenti, si veda Dorato (2013). 
mente corretta, "l'uso negativo porta i pantaloni" (Austin 1962a, p. 73). In assenza di un uso negativo, parlare di ciò che è reale è insensato. ${ }^{6}$

Per risolvere questa difficoltà concettuale, nel nostro caso la classe di contrasto tra chi nega e chi ammette la realtà dell'ora si potrebbe basare su un antico contrasto già messo in luce da Democrito: «Per convenzione si parla di colore, di dolce, di amaro; in realtà, ci sono soltanto atomi e vuoto». Democrito fr.125. La domanda nel nostro caso allora diviene: anche l'ora è per "convenzione"? Per convenzione qui Democrito intende, "dipendente dai nostri sensi", o meglio dall'incontro tra gli atomi e i nostri organi di senso. Sulla scia di questa distinzione, i filosofi corpuscolaristi dell'epoca moderna (Galileo, Cartesio, Gassendi, Locke, etc.) insistevano sulla differenza tra qualità primarie e secondarie. Le prime, numero e forma, permettevano di applicare la matematica al mondo oggettivo (aritmetica e geometria rispettivamente), mentre le seconde (sapore, odore, colore, suono) si dovevano lasciare alla dimensione qualitativa e soggettiva delle nostre menti. Per ciò che a noi interessa, affermare che l'ora è reale ed esiste indipendentemente da noi significa dire che "esser presente" è una proprietà intrinseca e non relazionale degli eventi fisici, ovvero non relativa a noi e ai nostri stati coscienti. ' Quindi se "l'ora" è reale nel senso di essere indipendente dalla mente, un evento sarebbe assolutamente e intrinsecamente presente anche se noi non ci fossimo. In una parola, stipuliamo che la proprietà "essere presente" è irreale se rappresenta una proiezione del soggetto sul mondo, e dunque è dipendente in questo senso dalla mente. Che una tesi filosofica realista in generale rimandi a "indipendenza da" è confermato anche da altri dibattiti intorno al realismo: il realismo sul mondo esterno è la sua esistenza indipendente dal suo essere percepito (contra Berkeley, "esse non est percipi"), mentre il realismo matematico afferma che una proposizione è vera indipendentemente da una dimostrazione o che le entità matematiche esistono indipendentemente da noi (platonismo matematico).

La seconda assunzione che farò è che le qualità primarie o ogget-

6 Esiste un altro modo per formulare il problema dell'esistenza del presente che non tratta esiste come un predicato ma qui non ne discuteremo.

7 Naturalmente, potrebbe essere relativa a un istante temporale, ma qui non si fa differenza tra evento e istante temporale. Questa nota va intesa come una risposta a un'obiezione di Matteo Morganti. 
tive siano quelle descritte dalla fisica (che dal suo punto di vista, come ebbe a scrivere Einstein a Schrödinger, è un tipo di metafisica), cosicché tali qualità sono reali nel senso chiarito sopra. Va però affrontata la domanda seguente: se la fisica attuale non descrive una qualsiasi proprietà $\mathrm{X}$, allora possiamo concludere che $\mathrm{X}$ è soggettiva o dipendente dalla mente? Nella letteratura filosofica, questa conclusione è stata spesso difesa, per esempio da Reichenbach nel uso famoso saggio "The Direction of Time": "se il divenire c'è, il fisico deve saperlo" (1956, p. 6) in ciò dando implicitamente per scontata la completezza epistemica della fisica, almeno in linea di principio.

\subsection{Realismi scientifici e realismi temporali}

Si potrebbe però obiettare che la distinzione tra proprietà primarie e secondarie, e quindi la formulazione stessa del realismo scientifico e del realismo temporale sull'ora in particolare, si basa su un controfattuale. Per l'antirealista la domanda "come sarebbe il mondo se noi non ci fossimo?" è insensata. Come facciamo infatti a fornire una descrizione del mondo senza le nostre facoltà cognitive, o meglio, come possiamo controllare la validità di una descrizione di $\mathrm{X}$ se $\mathrm{X}$ non è accessibile che tramite la nostra descrizione? Per difendere il realismo su un ente $\mathrm{X}$ o su una sua proprietà così come è descritto da una teoria scientifica, dovremmo accedere a X in modo "indipendente" dalla descrizione, come quando diciamo che un ritratto è somigliante a una persona che possiamo però percepire anche direttamente.

La radice di questa obiezione si nutre di un punto di vista "rappresentazionalista" alla conoscenza umana, secondo il quale conoscere il mondo esterno significa farsene un modello astratto che riproduca proprietà di esso utilizzando caratteristiche "visive" (vedi oltre). Tale punto di vista però contiene presupposizioni quantomeno discutibili, dato che il rapporto tra modello è mondo non è assimilabile a una riproduzione pittorica. Il modello è una costruzione umana, ma descrive aspetti del mondo che non dipendono da noi.

Per superare l'obiezione scettica di cui sopra, dapprima (i) conveniamo come sopra che se $\mathrm{X}$ è descritto dalla fisica come reale allora $\mathrm{X}$ è indipendente dalla mente; (ii) utilizziamo poi una o più delle quattro formulazioni di ciò che è reale da un punto di vista scientifico qui sotto descritte, e poi (iii) applichiamole al caso che ci interessa. Le formulazioni del realismo scientifiche possono schematizzarsi nel modo seguente. 
1. Realismo delle teorie.

2. Realismo delle entità (campi, particelle, stringhe...).

3. Realismo delle strutture (relazioni prioritarie su relata).

4. Realismo "debole" (fenomenico) $=$ intersoggettività. ${ }^{8}$

In ciò che segue, più che sviluppare in modo compiuto gli argomenti sollevati dalle quattro posizioni sopra elencate, le collegherò in modo sintetico alla grande tradizione filosofica che sta alla base del problema della realtà del tempo e del momento presente in particolare. Uno sviluppo più dettagliato di questa connessione deve essere lasciato a un lavoro futuro.

1. Il realismo sulle teorie evita l'obiezione strumentalista di cui sopra attraverso una virata in direzione semanticista: essere realisti sulle teorie spaziotemporali in questo primo senso implica affermare la verità approssimata delle teorie scientifiche e non invocare una forma pittorica o rappresentazioni delle teorie come "specchio della natura" (Rorty 1979). L'aggettivo "approssimata", definito in modo solo comparativo (le teorie quantitative della verisimiglianza soffrono di notevoli problemi) significa che, nel corso della storia della fisica, le teorie successive hanno inglobato importanti aspetti di quelle precedenti, riproducendo le previsioni di queste ultime in modo quantitativamente più preciso. Questo realismo sulle teorie, applicato a quelle spaziotemporali, implica per esempio che nella comparazione tra la teoria newtoniana e quelle relativistiche, le seconde siano più verosimili della prima, nel senso che sono più generali della prima e quindi più vicine alla teoria vera. Come già segnalato sopra, se reale significa indipendente dalla mente, questa presa di posizione realista naturalmente implica che il tempo di cui parla la fisica, nonché le sue proprietà, esistano indipendente da noi.

In assenza di ulteriori argomenti, si dovrebbe ammettere che la condizione (i) di cui sopra è solo sufficiente e non anche necessaria alla realtà di una caratteristica $\mathrm{X}$ del tempo. Come assunzione puramente concettuale la (i) appare ragionevole: è implausibile sostenere che il linguaggio fisico richieda un predicato X ("aver carica elettrica") per la formulazione delle sue leggi e delle sue descrizioni, senza dover assumere che tale predicato rimandi a una proprietà indipendente dalla mente umana (e.g., la carica elettrica). Il predicato e il concetto relativo serve solo a individuarla. Si può quindi concludere che se la fisica descrive

8 Per questa quadripartizione, si veda anche Dorato 1997. 
l'entità o la proprietà X con teorie approssimativamente vere, allora $\mathrm{X}$ è reale (esiste indipendentemente da noi). Tuttavia, senza un'assunzione molto controversa sulla completezza della fisica (la fisica attuale descrive tutto ciò che è reale) il realismo sulle teorie ci lascia in un'impasse. Contra Grünbaum (1963), il fatto che la fisica sinora abbia raggiunto un grande successo esplicativo senza presupporre l'esistenza un istante di tempo privilegiato (cioè la caratteristica X), non implica di per sé che il presente sia solo dipendente dalla nostra mente.

Detto in altro modo, dal punto di vista del "realista sull'ora", assumere il realismo sulle teorie scientifiche e su quelle spaziotemporali in particolare è solo una precondizione per rendere possibile la formulazione della sua tesi. Infatti, dando per scontato che

(a) la fisica non ha bisogno di una nozione privilegiata di presente per la formulazione delle sue leggi, visto che esse sono invarianti per traslazione temporale "in avanti e indietro" nel tempo a partire da qualunque istante;

(b) la relatività della simultaneità implica che il presente come attributo di un evento fisico o di un istante si riduca a un punto (Dorato 1995),

non ne segue che il presente sia soggettivo o dipendente dalla mente. Infatti

(c) dal realismo sulle teorie spaziotemporali non si può concludere né a favore del realismo né a favore dell'antirealismo sull'ora. La postulazione di un momento presente temporalmente privilegiato dipende da assunti metafisici che non possono essere confermati o supportati dalla fisica attuale, ma casomai solo dalla nostra esperienza soggettiva.

2. Il secondo tipo di realismo, quelle sulle cosiddette entità non osservabili o "teoriche", è assai interessante per i nostri scopi. I realisti e gli antirealisti sulle entità teoriche si dividono su ciò che esiste al di là di ciò che è osservabile direttamente. Entrambi ritengono che si può essere realisti sulle proprietà osservabili del mondo, ma solo i secondi credono nell'esistenza di quelle osservabili in modo indiretto (attraverso l'uso di strumenti), perché difendono una concezione della conoscenza di tipo interattivo e non "rappresentativo" (Hacking 1983). L'essere umano conosce quando interviene attivamente nel corso degli eventi interagendo con le sue proprietà causali. 
Nell'ambito di questa concezione dei rapporti tra fisica e mondo, il realista sulle entità può considerare il momento presente al pari di un'entità non osservabile direttamente. Come il tempo newtoniano, il momento presente sarebbe un'entità sostanziale in quanto esistente indipendentemente da altro e quindi anche da eventi fisici, e perciò ad essi non riducibile. La sua proprietà essenziale, per esprimersi ancora seguendo da vicino lo Scholium dei Philosopbiae Naturalis Principia Mathematica, sarebbe quella di "scorrere uniformemente". La tesi che il momento presente visto come una sostanza non sia osservabile direttamente non è incompatibile con il fatto che con la nostra azione e con le nostre osservazioni noi interagiamo indirettamente con eventi fisici che hanno la proprietà di essere presenti in quanto collocati in un particolare momento del tempo, ereditando dal tempo sostanziale questa proprietà. La reificazione del tempo visto come entità indipendente da qualunque altra sostanza o entità tuttavia genera tuttavia notevoli difficoltà, dato che implica che il tempo possa esistere senza il campo gravitazionale, una implicazione che alla luce della relatività generale appare poco plausibile. Anche in questo caso, il realista metafisico sull'ora deve assumere che esista qualche tipo di entità metafisica non osservabile direttamente che può essere postulata solo oltrepassando la conoscenza fisica e addirittura creando un conflitto con essa.

3. Il realismo sulle strutture o realismo strutturale, oggi al centro del dibattito in filosofia della fisica (Ladyman e Ross 2011) sostiene che le relazioni che intercorrono tra entità sono epistemicamente ed ontologicamente fondamentali rispetto ai relata. Non solo le relazioni sono ciò che ci permette di conoscere (realismo epistemico) i relata ma questi ultimi traggono la loro identità dalle prime e in questo senso sono onticamente più fondamentali.

Questo tipo di realismo scientifico, se declinato in funzione del nostro problema, implica una concezione relazionista del tempo. In tale concezione, il tempo è l'ordine di successione tra eventi e le relazioni temporali non esisterebbero senza eventi fisici, che è tipicamente associata alla posizione di Leibniz.' Gli instanti temporali non esistono perché

9 In realtà lo studio dei manoscritti inediti di Newton ha mostrato che quest'ultimo è assai meno sostanzialista di quanto la letteratura del positivismo logico ci abbia fatto credere. 
sono privi di identità intrinseca e hanno quindi una natura puramente relazionale. Se il tempo è essenzialmente relazionale, l'attribuzione della proprietà temporale apparentemente intrinseca "essere presente" implicherebbe di fatto l'attribuzione di una relazione o a coppie di eventi fisici o a coppie di istanti temporali. Un evento fisico $\mathrm{E}$ (un istante) può essere presente solo se entra in una relazione $\mathrm{R}$ rispetto a un altro evento $\mathrm{F}$ (un altro istante). Ma quale relazione? Dato che $\mathrm{R}=$ 'essere presente' è transitiva simmetrica e riflessiva, $R$ è una relazione di equivalenza e le classi sono presumibilmente formate da eventi tra loro simultanei, cosicché "essere presente" e "essere simultaneo" sono co-estensionali.

Proprio questa identificazione estensionale genera un attrito concettuale con la relatività della simultaneità impostaci della relatività speciale. In questa teoria, quel che conta come simultaneo (e quindi presente) è dipendente dall'arbitraria scelta di un sistema di riferimento inerziale. In relatività un evento può quindi presente solo rispetto a se stesso, ciò che rende il presentismo, la tesi che esiste solo ciò che esiste nel presente "solipsistica", e quindi tale da dover spiegare perché sembriamo condividiamo l' "ora" (ma non il "qui"). Una concezione relazionista sull'ora che postuli la sua realtà implica la postulazione di una superficie di simultaneità privilegiata che, dopo Einstein, la fisica non può descrivere nemmeno in linea di principio.

Per comprendere il senso di questa conclusione da un'altra angolatura, a causa della relatività della simultaneità l'esistenza di una classe di eventi simultanei dipende dalla scelta di un arbitrario sistema di riferimento inerziale, cosicché gli eventi presenti sono essi stessi relativi a tali sistemi. Siccome la simultaneità tra eventi vale in modo assoluto o invariante solo in una zona ristretta dello spaziotempo (a rigore puntiforme), lo stesso vale per la nozione di presente: il presente si riduce a un punto o poco più, e quindi la tesi che esista solo il presente, intesa come una fedele riproduzione della nostra esperienza del tempo viene ridotta all'assurdo: per ogni punto nello spazio e nel tempo, è presente solo quel punto e nessun'altro.

Come per il realismo delle teorie, anche nel caso di concezione relazionista del tempo di tipo 'leibniziano' la realtà del momento presente dipende da un'assunzione metafisica che per il momento può essere giustificata solo sulla base della nostra esperienza soggettiva. Poiché essa sembra andare contro quel che affermano le teorie fisiche più confermate, raggiungiamo anche in questo caso la stessa conclusione scettica che avevamo sottolineato a proposito del realismo sulle teorie. 
4. L'ultima posizione realistica sulla scienza è quella "debole" o kantiana, in base alla quale la conoscenza scientifica non coglie la realtà in sé come afferma il realismo metafisico. Piuttosto, essa coglie i fenomeni in modo intersoggettivamente valido. Rispetto a questi ultimi, il tempo è empiricamente reale, ovvero ogni giudizio temporale è intersoggettivamente condiviso perché fondato sulla categoria della causalità, ma tale categoria ${ }^{10}$ può fornire conoscenza solo se applicata ai fenomeni. I fenomeni sono ciò che le nostre strutture trascendentali rendono conoscibile rispetto a un oggetto inconoscibile ma pensabile, che possiamo con un certo grado di approssimazione chiamare "cosa in sé", o la realtà metafisica in sé. Se riferito alla realtà in sé però, il tempo visto come intuizione è puramente ideale. Per Kant, non intuiamo il tempo come proprietà metafisica del mondo. Da un punto di vista trascendentale, il presente è visto come l'attribuzione intersoggettivamente valida perché condivisa di una certa proprietà agli istanti temporali, così come sono sintetizzati a priori dalla nostra coscienza (o meglio, per essere più precise, dal nostro "io penso"). Dal punto di vista della filosofia kantiana quindi, il presente è empiricamente ma non metafisicamente reale, dato che non può essere attribuito o predicato della cosa in sé. Ma poiché abbiamo stipulato che una proprietà è reale se e solo se esisterebbe anche se la nostra mente non la concettualizzasse o la intuisse, nostro punto di vista, l'ora come lo concettualiza Kant è irreale perché dipendente dalla mente.

Ricapitolando le conseguenze filosofiche della quadripartizione di cui sopra per il tema di questo saggio: il presente appare al massimo una postulazione metafisica che non ha supporto diretto dalla fisica, anche se quest'ultima non la può escludere. Nella prossima sezione vedremo in effetti come il flusso del tempo nasca dall'interazione tra successioni locali di eventi descrivibili dalla fisica con successioni di percezioni soggettive coscienti integrate dalla consapevolezza della loro successione.

\section{IL CARATTERE RELAZIONALE DEL PRESENTE E IL SUO PASSAGGIO}

Il fatto che la non descrivibilità del presente da parte della fisica

10 Se si sostituisce il termine desueto "categoria" con schema concettuale o semplicemente concetto, possiamo dire che la causalità intesa come schema concettuale ordina i fenomeni fisici attraverso regolarità ripetibili. 
non implichi l'illusorietà del suo "passare" può essere argomentato con le tre osservazioni seguenti.

La prima sviluppa le riflessioni concettuali già esposte più sopra. Anzitutto l'illusione è un fenomeno percettivo che si distingue da uno non illusorio grazie a caratteristiche ben delineabili. Il tipico esempio del remo spezzato rimanda a due descrizioni o rappresentazioni percetti$v e$ in conflitto, $\mathrm{D}_{\mathrm{R}}$ e $\mathrm{D}_{\mathrm{A}}$. Da una parte, $\mathrm{D}_{\mathrm{R}}$ riguarda la descrizione del remo fisico data dall'evidenza tattile - che è in ogni caso epistemicamente indipendente da quella visiva - e dalla successiva estrazione del remo dall'acqua: queste due evidenze sensoriali ci convincono del fatto che il remo non è "realmente" o "veramente" spezzato ma solo apparentemente. L'altra descrizione $\mathrm{D}_{\mathrm{A}}$ contiene la rappresentazione del remo immerso nell'acqua che chiamiamo illusoria: in questa seconda rappresentazione il remo appare davvero spezzato alla nostra visione per ragioni fisiche che ben conosciamo e che dipendono dall'interazione tra aria, acqua (rifrazione) luce e apparato visivo.

In questo caso, come in altri di questo tipo, il carattere illusorio della percezione è basato sulla conoscenza di ciò che significa affermare che il remo non è spezzato, che è ciò che si percepisce in condizioni fisiche diverse. Nel caso dell'esperienza del passaggio del tempo, non riusciamo a indicare come potrebbe essere la nostra esperienza se nella nostra coscienza non si succedessero percezioni di eventi fisici - a loro volta in successione -che vengono poi integrate in un unico atto di coscienza che genera la consapevolezza del loro essere successive. Una successione di esperienze coscienti non basta infatti per la consapevolezza della loro successione.

La seconda osservazione è anch'essa stimolata dall'esempio appena fatto: quando affermiamo che un'esperienza è illusoria dobbiamo essere in grado di spiegare il sorgere dell'illusione a partire da un insieme di situazioni non illusorie. Come noto, questo compito esplicativo è stato realizzato dai filosofi copernicani quando spiegarono perché la Terra si muove rispetto al centro di massa Terra-Sole anche se noi non ce ne accorgiamo. Malgrado alcuni tentativi da parte di fisici teorici che hanno provato a spiegare la nostra esperienza del tempo a partire da una realtà fisica atemporale (Barbour 1999), i tentativi finora fatti non sembrano soddisfacenti.

La terza parte da un dato della nostra esperienza difficilmente confutabile perché basato sulla nostra natura biologica. Noi anticipiamo ciò che sta per accedere sia a brevissima scadenza - come quando 
istintivamente e automaticamente evitiamo un oggetto che ci è stato tirato contro - sia a intervalli temporali più remoti nel futuro, come quando pianifichiamo il lavoro da svolgere il giorno successivo, la settimana successiva o addirittura l'anno successivo. Il "tempo di attesa" di cui sopra è dovuto alle nostre capacità anticipatorie e predittive, che nella nostra specie sono assai più sviluppate che in qualunque altra. Anticipiamo ciò che accadrà nella prossima domenica sulla base delle esperienze passate, anticipiamo i giorni della vecchiaia con maggiore angoscia perché vediamo la sorte che tocca alle persone anziane accanto a noi. La credenza che la distanza temporale tra presente e evento anticipato diminuisca sempre più non può essere sradicata da nessuna teoria o ipotesi fisica. La giustificazione più forte di questa credenza è che quando la separazione temporale tra evento presente e evento futuro anticipato è minima, dipende da una percezione immediata della successione che è non inferenziale. Si noti che si può afferamre con sicurezza che uno degli eventi esperiti $E$ risulta causalmente precedente rispetto alla nostra percezione di esso, secondo la relatività speciale la distinzione temporale tra ciò che precede l'esperienza di $E$ e ciò che la segue è oggettiva e invariante per ogni osservatore.

Considerando la nostra credenza che il tempo passi come non sradicabile da ipotesi teoriche che vengono dalla fisica, è importante interrogarsi sulle condizioni che la rendono possibile. Una opzione è che il passaggio del tempo sia un caratteristica fondamentale dell'universo fisico e quindi da considerarsi un presupposto necessario della descrizione fisica del mondo. ${ }^{11}$ Un'altra possibilità, che personalmente prediligo, è provare a spiegarlo tenendo conto di due ingredienti. In primis deve esistere una successione oggettiva e locale ${ }^{12}$ di eventi fisici, che è il passaggio del tempo minimale di cui può parlare la fisica. Il passaggio del tempo è minimale perché nessun evento fisico nella successione può contare come presente se non in relazione a noi. Ma tale relazione è oggettiva perché non illusoria, benché non sia riducibile alla fisica e sia dipendente dalla mente. ${ }^{13}$ Infatti gli eventi della successione fisica ven-

11 Questa teoria è difesa in Maudlin (2010).

12 La successione fisica è locale perché solo in questo caso non si generano contraddizioni con la richiesta della teoria della relatività, che afferma che il presente e la simultaneità sono oggettivi solo se "quasi puntiformi".

13 Nel senso di reale dato da "dipendente dalla mente" il presente non è reale. 
gono prima anticipati, poi vissuti e infine rammemorati. Questo è il secondo ingrediente di cui sopra. È la differente esperienza psicologica che accompagna questi tre atteggiamenti temporali così come essa si riferisce alla successione fisica di eventi che può costituire un primo nucleo esplicativo del nostro senso del passaggio del tempo.

Ogni nostra esperienza per definizione avviene nel presente, ovvero, ogni volta che facciamo esperienza di un evento $E$ lo percepiamo nel presente perché avvertiamo la differenza tra la percezione diretta di $E$, il ricordo di un evento $F$ esperito prima di $E$ avvenuto nel passato, e ovviamente anche la differenza tra $E$ e un evento $G$ che anticipiamo mentre "viviamo" $E$. In termini Humeani, le percezioni immediate sono più "vivide". Senza presupporre questa tripartizione di atteggiamenti temporali, la nostra attività cognitiva, inclusa quella che rende possibile la fisica, sarebbe impossibile o quantomeno incomprensibile. Ma tale carattere "trascendentale" della costruzione umana del tempo non implica che la descrizione fisica e oggettiva dell'universo necessiti di un momento presente privilegiato.

Fatte queste dovute osservazioni, che rimandano al carattere indubitabile di alcune caratteristiche essenziali del tempo della nostra esperienza, occorre ricordare che esistono aspetti della nostra visione ingenua del tempo che sono stati "corretti" dall'evoluzione del pensiero fisico. Tra questi in particolare la credenza che il presente si estenda a distanza, fatta propria, come il lettore ricorderà dalla citazione di cui sopra, persino da Newton. Già solo questo fatto dimostra quanto questa credenza sia profondamente radicata nel nostro modo di costruire il mondo a partire dal linguaggio ordinario e dal nostro apparato cognitivo.

La credenza che ciò che è percepito nel presente si estenda a distanze cosmiche dipende dal fatto che confondiamo "percepito simultaneamente" e "avvenuto simultaneamente"; la percezione dell'esplosione della supernova avviene contemporaneamente a quella dell'albero che in parte la nasconde alla nostra vista ma l'intervallo di tempo che separa l'emissione della luce che arriva alle nostre retine da quella relativa all'albero è molto grande e può corrispondere a vari anni luce: "The illusion which prevailed prior to the enunciation of the theory of relativity ... had its origin in the fact that in our everyday experience we can neglect the time of propagation of light. We are accustomed on this account to fail to differentiate between "simultaneously seen" and "simultaneously happening"; and, as a result, the difference between time and local time is blurred." (Einstein 1936, p. 
299). Il tempo (time) cui si riferisce Einstein in questa citazione è il tempo fissato dalla simultaneità tra i due eventi di emissione che è relativa al nostro sistema di riferimento ed è quindi quel che si chiama tempo coordinato. Il tempo locale (local time) è quello che coinvolge la percezione simultanea dei due eventi da parte delle nostre retine, che è locale perché avviene in un intorno molto piccolo dello spaziotempo.

Ne segue quindi che è solo un aspetto delle nostre credenze relative al presente che deve essere abbandonato e corretto dalle nostre conoscenza fisiche, ovvero il carattere cosmico del presente. Si badi però al fatto che la nostra credenza nel presente esteso è giustificata dalla velocità praticamente istantanea della luce e dalle soglie dei nostri tempi percettivi, che per quanto piccole, non sono infinitesime. Per percepire due segnali visivi come distinti abbiamo bisogno di un intervallo di almeno $40 \mathrm{~ms}$. In $40 \mathrm{~ms}$ la luce percorre $12000 \mathrm{~km}(=300.000 x 30 \mathrm{~ms})$ e nessuna coppia di segnali luminosi temporalmente separati dal punto di vista fisico ma contenuto entro il raggio di quella sfera può essere percepita come caratterizzata da una successione temporale. Analogamente a quando si riteneva che la terra fosse un disco piatto a causa del suo grande raggio $^{14}$ e a quando si riteneva lo spazio-tempo piatto a causa del campo gravitazionale debole nel quale viviamo, anche in questo caso, prima di Einstein, abbiamo proiettato la nostra esperienza locale del presente a tutto l'universo. Ma come questi importanti esempi storici mostrano, gli errori compiuti dal senso comune non sono illusioni, ma piuttosto approssimazioni più o meno fedeli alle teorie fisiche.

\section{BIBLIOGRAFIA}

Austin J. (1962) Sense and Sensibilia, Oxford University Press. Barbour J. (1999), The end of time, Oxford University Press, Oxford, 1999.

Carnap, R. (1963) "Carnap's Intellectual Biography" in The Philosophy of Rudolf Carnap, P. A. Schilpp (ed.), pp. 3-84. Open Court, La Salle, IL.

Dorato M. (1995), Time and Reality, Clueb, Bologna.

Dorato M, (2008), Cosa c'entra l'anima con gli atomi, Laterza, Roma.

Dorato M. (2013), Che cos'è il tempo? Carocci, Roma.

14 La curvatura $\mathrm{C}$ di una sfera è inversamente proporzionale al quadrato del suo raggio $\mathrm{r}, \mathrm{C}=1 / \mathrm{r}^{2}$, così che tanto maggiore è il raggio della sfera tanto più piccola è la sua curvatura. Se il raggio tende all'infinito la curvatura di una superficie è nulla. 
Einstein, A. (1936) "Physik und Realität”, Journal of The Franklin Institute 221: 313347, English translation in Ideas and Opinion, Bonanza, New York, 1936.

Grünbaum A. (1963), Philosophical Problems of Space and Time. Reidel Dordrecht, Boston.

Gunn A. J. (1929) The problem of time. An historical and critical study, George Allen \& Unwin Ltd. London.

Hacking I. (1983), Representing and Intervening. Cambridge University Press, Cambridge.

Ladyman J. Ross D. 2007 Everything must go, Oxford University Press, Oxford.

Maudlin, T. (2010), "On the Passage of Time" in The Metaphysics within Physics. Oxford University Press, pp. 104-142.

Reichenbach H. (1956) The Direction of Time. Berkeley University Press, Berkeley.

Rorty R. (1979) Philosophy and the Mirror of nature, Princeton University Press, Princeton.

Ruggiu, L. (2008) (a cura di) La fisica. Testo greco a fronte. Mimesis, Roma.

Skow B. (2015), Objective Becoming. Oxford University Press, Oxford.

Torretti, R. (2006), Can science advance effectively through philosophical criticism and reflection? [Preprint], in http://philsci-archive.pitt.edu/2875/.

Torretti R. (2007) The Problem of the time's arrow historico-critical reexaminated, Studies in History and Philosophy of Modern Physics : 38, 732-756. 